\title{
Does surgical approach affect patient outcomes of total knee arthroplasty?
}

\author{
Sahil Prabhnoor Sidhu, MD \\ Lyndsay E. Somerville, PhD \\ Aamir Sohail Sidhu, BSc \\ Ryan T. Willing, PhD \\ Matthew G. Teeter, PhD \\ Brent A. Lanting, MD
}

Accepted Nov. 5, 2020

\section{Correspondence to:}

S.P. Sidhu

339 Windermere $\mathrm{Rd}$

London ON N6A 5A5

prabhnoor.sidhu@ucalgary.ca

Cite as: Can J Surg 2021 October 1; 64(5). doi: 10.1503/cjs.010920
Background: Surgical approaches for total knee arthroplasty (TKA) include the medial parapatellar (MPA), subvastus (SV), midvastus (MV), and lateral parapatellar approach (LPA); it remains unclear which approach is superior.

Methods: Patients having undergone TKA at our institution were retrospectively organized into matched groups according to surgical approach (MPA, MV, SV, or LPA). Outcomes between the groups were compared using the Short-Form 12 (SF-12), Western Ontario and McMaster Universities Osteoarthritis Index (WOMAC), Knee Society Score (KSS), and range of motion (ROM) up to 2 years postoperative.

Results: Sixty-eight MV patients, $8 \mathrm{SV}$ patients, and $4 \mathrm{LPA}$ patients were matched with groups of MPA patients. There was no difference in outcomes between the MPA and MV groups up to 2 years. The SV group had significantly higher SF-12 Physical Composite Score (PCS; $p=0.036)$ and WOMAC stiffness score $(p=$ $0.014)$ at 2 years, but significantly lower flexion at 1 year $(p=0.022)$ than the MPA group. The LPA group had significantly lower SF-12 PCS $(p=0.011)$ and WOMAC function scores $(p=0.022)$ at 1 year than the MPA group.

Conclusion: There was no significant difference between the MPA and MV approach. The SV approach had some improved long-term outcomes over the MPA aproach (SF-12 and WOMAC), but had significantly lower flexion at 1 year. The LPA group showed inferior outcomes than the MPA group but had more severe valgus preoperative deformity $(p=0.024)$. Further studies are required to investigate the potential benefit of quadriceps-sparing approaches.

Contexte : Les voies chirurgicales d'arthroplastie totale du genou (ATG) sont les suivantes : parapatellaire interne (PI), subvastus (SV), midvastus (MV), et parapatellaire externe (PE); il n'est pas clair quelle voie est supérieure.

Méthodes : Les patients qui ont subi une ATG dans notre établissement ont été classés rétrospectivement en groupes appariés selon la voie chirurgicale (PI, MV, $\mathrm{SV}$ ou $\mathrm{PE}$ ). Les résultats des différents groupes ont été comparés au moyen du Short-Form 12 (SF-12), de l'indice Western Ontario and McMaster Universities Osteoarthritis Index (WOMAC), du score de la Knee Society (KSS) et de l'amplitude du mouvement jusqu'à 2 ans après l'opération.

Résultats : Au total, 68 patients traités par voie $M V, 8$ par voie SV et 4 par voie $\mathrm{PE}$ ont été appariés avec des groupes de patients traités par voie PI. Il n'y avait aucune différence dans les résultats entre les groupes PI et MV jusqu’à 2 ans. Comparativement au groupe PI, le groupe SV avait des résultats significativement plus élevés pour le score physique fonctionnel (PCS) du SF-12 $(p=0,036)$ et le score de raideur de l'indice WOMAC $(p=0,014)$ après 2 ans, mais une flexion significativement plus faible après 1 an $(p=0,022)$. Encore comparativement au groupe PI, le groupe PE avait des résultats significativement plus faibles pour le PCS du SF-12 $(p=0,011)$ et les scores fonctionnels de l'indice WOMAC $(p=0,022)$ après 1 an.

Conclusion : Il n'y avait pas de différence significative entre les voies PI et MV. $\mathrm{La}$ voie $\mathrm{SV}$ offrait de meilleures issues à long terme que la voie PI (SF-12 et WOMAC), mais une flexion significativement plus faible après 1 an. Le groupe $\mathrm{PE}$ a eu des issues moins bonnes que le groupe PI, mais présentait plus souvent une déformation valgus préopératoire grave $(p=0,024)$. D'autres études seront requises pour établir les bienfaits potentiels de voies évitant le quadriceps. 
$\mathbf{M}$ any approaches have been described for total knee arthroplasty (TKA), including the medial parapatellar (MPA), subvastus medialis (SV), midvastus (MV), and lateral parapatellar (LPA) approaches. The most commonly used of these is the MPA, which is considered the standard to which others are compared.

In a standard MPA, a midline incision is used and a medial parapatellar arthrotomy is made. It allows for excellent exposure and is relatively straightforward to perform. ${ }^{1}$ An SV approach typically also involves a midline skin incision, but the incision may be positioned more oblique and medially. From there, the border of the vastus medialis is visualized, its fascia is incised, and the vastus medialis is bluntly elevated from the medial intermuscular septum..$^{1,2}$ Engh and colleagues described a modification to the SV approach in which the vastus medialis is split in line with its muscle fibres proximally; this was named the MV approach. ${ }^{3}$ Finally, the LPA also involves a midline incision, but may be positioned lateral to the tibial tubercle. The arthrotomy is made lateral to the patella ${ }^{4}$ and extends into the quadriceps tendon, leaving a small lateral margin to enable repair.

Each approach has advantages and disadvantages. For example, although the MPA provides an excellent view of the joint, it involves violating the extensor mechanism and medial structures. ${ }^{1}$ The $\mathrm{SV}$ and $\mathrm{MV}$ approaches are "quadriceps sparing," but are more technically difficult to perform and often reserved for thinner patients. ${ }^{1,3,5-8}$ They may also result in decreased accuracy of implant positioning. ${ }^{9}$ The LPA provides direct access to lateral structures in valgus knees and spares medial soft tissues, but also can be technically challenging. ${ }^{1,4}$ It has also been described in conjunction with a tibial tubercle osteotomy (TTO).

Although several randomized controlled trials exist comparing the SV and MV approaches to the MPA, sample sizes are small and the results are mixed. For example, Cho and colleagues ${ }^{10}$ found results favouring the MV approach; Heekin and colleagues ${ }^{11}$ and Pan and colleagues ${ }^{12}$ found no significant difference between the approaches; Varela-Egocheaga and colleagues ${ }^{13}$ found results favouring the SV approach; and Varnell and colleagues ${ }^{14}$ found results favouring the MPA.

Similarly, RCTs comparing the lateral and medial parapatellar approaches also tend to have small sample sizes and report mixed results. Many studies also incorporate use of a TTO, which makes results difficult to interpret. Nonetheless, several studies have reported promising outcomes with this approach in valgus knees. ${ }^{15,16}$

Given the small sample size and mixed results of existing studies, we aimed to add to the body of literature by retrospectively reviewing patients at our institution and comparing outcomes of the SV, MV, and LPA to the standard MPA.

\section{Methods}

After obtaining approval from our institutional ethics board, we searched the institutional database for patients having undergone primary TKA between 2015 and 2019. Patients were included if they were older than 30 years and underwent primary TKA for osteoarthritis. Revision cases were excluded, as were patients who underwent arthroplasty for a reason other than osteoarthritis. All TKAs were performed by a single surgeon at our institution, with the vast majority of cases using a Triathlon implant (Stryker).

Patient demographic characteristics were recorded. Short-Form 12 (SF-12), Western Ontario and McMaster Universities Osteoarthritis Index (WOMAC), Knee Society Score (KSS), knee function scores, and range of motion (ROM) were recorded at yearly intervals from the initial surgical date. The WOMAC scores were reported on a scale of 0 (worst) to 100 (best). Any instance of revision, as well as the reason for revision, were also tracked.

Charts were retrospectively reviewed by the lead author in order to organize patients by surgical approach used. The MV and SV approaches were generally chosen to offer a potentially less invasive approach to patients with varus or neutral alignment and lower body mass index (BMI). The LPA was used in cases of severe valgus deformity, as it is the lead surgeon's preferred approach for such cases. Patient groups were matched using propensity score matching for age, BMI, and gender.

\section{Statistical analysis}

Statistical analysis between groups was performed using SPSS Statistics version 23 (IBM). We used the Student $t$ test to compare the cohorts; the Welch $t$ test was used in cases when we were unable to assume similar variance.

\section{Results}

\section{Medial parapatellar v. midvastus approach}

After matching for age and BMI, 68 patients met the inclusion criteria for the medial parapatellar group, with a mean age of $66.7 \pm 9.6$ years and a mean BMI of $30.5 \pm$ $4.8 \mathrm{~kg} / \mathrm{m}^{2}$. Forty-eight $(70.6 \%)$ of the MPA patients were male and $20(29.4 \%)$ were female. Sixty-eight patients were included in the MV group, with a mean age of $68.0 \pm$ 9.2 years and a mean BMI of $30.8 \pm 4.7 \mathrm{~kg} / \mathrm{m}^{2}$. Twentynine $(42.7 \%)$ of the MV patients were male and 39 $(57.4 \%)$ were female. There was no significant difference in age $(p=0.426)$ or BMI $(p=0.776)$ between these groups. There was a significant difference in the gender make-up of these groups $(p=0.001)$. Of the patients who underwent an MPA, 29 had a final follow-up up to 1 year postoperative and 39 had a final follow-up up to 2 years 
postoperative. In the MV group, 49 patients had a final follow-up up to 1 year postoperative and 19 had a final follow-up up to 2 years postoperative. Sixty $(88.2 \%)$ of the MPA patients and 59 (86.8\%) of the MV patients had preoperative varus alignment.

Outcome scores of the MPA and MV groups are summarized in Table 1 . There were no significant differences up to 2 years between these 2 groups.

Of the MPA patients, 2 (2.9\%) required revision; 1 patient underwent revision for lateral patellar subluxation and the other had patellar resurfacing for anterior knee pain. Three (4.4\%) patients in the MV group required revision; 1 patient underwent revision for aseptic loosening of the tibial component and 2 patients underwent patellar resurfacing for anterior knee pain.

\section{Medial parapatellar v. subvastus medialis approach}

After matching for age and BMI, 8 patients met the inclusion criteria for the MPA group, with a mean age of $68.2 \pm$ 8.8 years and a mean BMI of $28.4 \pm 1.9 \mathrm{~kg} / \mathrm{m}^{2}$. One $(12.5 \%)$ patient in the MPA group was male and 7 $(87.5 \%)$ were female. Eight patients were included in the SV group, with a mean age of $70.4 \pm 6.5$ years and a mean BMI of $28.5 \pm 2.3 \mathrm{~kg} / \mathrm{m}^{2}$. Three $(37.5 \%)$ patients in the SV group were male and $5(62.5 \%)$ were female. There was no significant difference in age $(p=0.575), \mathrm{BMI}(p=$ $0.914)$, or gender make-up $(p=0.248)$ between these groups. In the MPA group, all 8 patients had a final follow-up up to 2 years postoperative. In the SV group, 2 patients had a final follow-up up to 1 year postoperative and 6 patients had a final follow-up up to 2 years postoperative. All patients had preoperative varus alignment in both groups.

Outcome scores between the MPA and SV groups are summarized in Table 2. There was a significant difference in SF-12 Physical Composite Score (PCS) at 2 years postoperative $(p=0.036)$ and in the WOMAC stiffness score at 2 years postoperative $(p=0.014)$, both favouring the SV approach. The MPA group had significantly higher flexion at 1 year postoperative $(p=0.022)$. There were no other significant difference between the 2 approaches at other time points, as summarized in Table 3 .

One patient $(12.5 \%)$ in the MPA group required revision in the form of patellar resurfacing for anterior knee pain. No patients in the SV group underwent revision.

\section{Medial parapatellar v. lateral parapatellar approach}

As the LPA is generally reserved for valgus knees, only knees with valgus preoperative alignment in each group were compared. The matched valgus MPA group had a mean age of $69.0 \pm 12.3$ years and a mean BMI of $30.5 \pm$ $0.4 \mathrm{~kg} / \mathrm{m}^{2}$. The valgus LPA group had a mean age of $69.6 \pm 21.1$ years and a mean BMI of $31.4 \pm 6.9 \mathrm{~kg} / \mathrm{m}^{2}$.
Table 1. Outcome scores in patients who underwent medial parapatellar and midvastus approaches, up to 2 years postoperative

\begin{tabular}{|c|c|c|c|}
\hline \multirow[b]{2}{*}{ Outcome } & \multicolumn{2}{|c|}{ Group; mean \pm SD } & \multirow[b]{2}{*}{$p$ value } \\
\hline & MPA & MV & \\
\hline SF-12 MCS, 1 yr postop & $52.3 \pm 10.4$ & $52.3 \pm 10.9$ & 0.79 \\
\hline SF-12 MCS, 2 yr postop & $53.6 \pm 9.2$ & $54.9 \pm 9.7$ & 0.69 \\
\hline SF-12 PCS, 1 yr postop & $41.0 \pm 10.1$ & $44.0 \pm 10.4$ & 0.12 \\
\hline SF-12 PCS, 2 yr postop & $32.4 \pm 10.5$ & $42.5 \pm 10.3$ & 0.95 \\
\hline WOMAC pain, 1 yr postop & $80.0 \pm 20.7$ & $81.4 \pm 18.2$ & 0.71 \\
\hline WOMAC pain, 2 yr postop & $82.9 \pm 16.8$ & $81.1 \pm 32.9$ & 0.86 \\
\hline WOMAC stiffness, 1 yr postop & $70.4 \pm 24.1$ & $72.1 \pm 19.4$ & 0.68 \\
\hline WOMAC stiffness, 2 yr postop & $79.1 \pm 18.8$ & $71.1 \pm 26.8$ & 0.28 \\
\hline WOMAC function, 1 yr postop & $78.5 \pm 20.2$ & $78.9 \pm 18.3$ & 0.92 \\
\hline WOMAC function, 2 yr postop & $82.6 \pm 18.1$ & $83.2 \pm 21.9$ & 0.93 \\
\hline WOMAC total, 1 yr postop & $77.8 \pm 19.7$ & $78.9 \pm 16.6$ & 0.78 \\
\hline WOMAC total, 2 yr postop & $81.8 \pm 15.6$ & $83.3 \pm 21.3$ & 0.81 \\
\hline Extension, ${ }^{\circ}, 1$ yr postop & $0.2 \pm 1.4$ & $0.5 \pm 1.8$ & 0.31 \\
\hline Extension, ${ }^{\circ}, 2$ yr postop & $0.7 \pm 1.8$ & $0.8 \pm 1.9$ & 0.82 \\
\hline Flexion, $\stackrel{\circ}{, 1}$ yr postop & $119.4 \pm 10.3$ & $114.7 \pm 19.3$ & 0.13 \\
\hline Flexion, ${ }^{\circ}, 2$ yr postop & $120.2 \pm 9.6$ & $114.6 \pm 11.2$ & 0.13 \\
\hline KSS function, 1 yr postop & $83.2 \pm 21.2$ & $85.2 \pm 18.9$ & 0.61 \\
\hline KSS function, 2 yr postop & $85.0 \pm 19.8$ & $90.9 \pm 12.2$ & 0.29 \\
\hline KSS knee, 1 yr postop & $91.5 \pm 11.6$ & $93.0 \pm 9.8$ & 0.50 \\
\hline KSS knee, 2 yr postop & $91.4 \pm 13.7$ & $90.6 \pm 12.8$ & 0.87 \\
\hline KSS total, 1 yr postop & $173.5 \pm 28.9$ & $176.3 \pm 25.6$ & 0.63 \\
\hline KSS total, 2 yr postop & $174.4 \pm 31.6$ & $184.4 \pm 19.0$ & 0.28 \\
\hline \multicolumn{4}{|c|}{$\begin{array}{l}\text { KSS = Knee Society Score; MPA = medial parapatellar approach; MV = midvastus } \\
\text { approach; SF-12 MCS = Short-Form } 12 \text { Mental Composite Score; SF-12 PCS = } \\
\text { Short-Form } 12 \text { Physical Composite Score; WOMAC = Western Ontario and MacMaster } \\
\text { Universities Osteoarthritis Index. }\end{array}$} \\
\hline
\end{tabular}

All patients in both groups were female. There was no significant difference in age $(p=0.96), \mathrm{BMI}(p=0.83)$, or gender make-up between the groups. All patients had a final follow-up up to 1 year postoperative. The mean preoperative valgus angle in the LPA group was $17.4^{\circ} \pm$ $5.1^{\circ}$, which was significantly higher than the mean valgus angle of $7.0^{\circ} \pm 4.7^{\circ}$ in the MPA group ( $\left.p=0.024\right)$.

Outcome scores for each group are summarized in Table 3. There was a significant difference in SF-12 PCS at 1 year postoperative $(p=0.011)$ and in WOMAC function score at 1 year postoperative $(p=0.022)$, both favouring the MPA. There was no significant difference in SF-12 Mental Composite Score (MCS), WOMAC pain, stiffness, and total scores, ROM, or KSS at 1 year postoperative.

No patients in either group required revision.

\section{Discussion}

Although there are several randomized controlled trials comparing the MPA, MV, SV, and LPA approaches, many are small in sample size, report mixed results, and have short follow-up duration. ${ }^{16,17} \mathrm{We}$ aimed to add to this body of literature by reporting outcomes of a singlesurgeon, expertise-based study. 
Table 2. Outcome scores in medial parapatellar and subvastus approach patients up to 2 years postoperative

\begin{tabular}{|c|c|c|c|}
\hline \multirow[b]{2}{*}{ Outcome } & \multicolumn{2}{|c|}{ Group; mean $\pm S D$} & \multirow[b]{2}{*}{$p$ value } \\
\hline & MPA & SV & \\
\hline SF-12 MCS, 1 yr postop & $56.7 \pm 11.5$ & $58.0 \pm 2.3$ & 0.82 \\
\hline SF-12 MCS, 2 yr postop & $53.7 \pm 10.3$ & $52.2 \pm 8.8$ & 0.82 \\
\hline SF-12 PCS, 1 yr postop & $45.4 \pm 13.2$ & $43.1 \pm 13.1$ & 0.77 \\
\hline SF-12 PCS, 2 yr postop & $38.6 \pm 11.5$ & $53.2 \pm 5.1$ & 0.036 \\
\hline WOMAC pain, 1 yr postop & $71.4 \pm 26.3$ & $86.4 \pm 19.3$ & 0.25 \\
\hline WOMAC pain, 2 yr postop & $72.5 \pm 20.6$ & $98.0 \pm 2.7$ & 0.09 \\
\hline WOMAC stiffness, 1 yr postop & $62.5 \pm 29.8$ & $75.0 \pm 21.6$ & 0.39 \\
\hline WOMAC stiffness, 2 yr postop & $56.3 \pm 12.5$ & $85.0 \pm 13.7$ & 0.014 \\
\hline WOMAC function, 1 yr postop & $74.1 \pm 25.2$ & $84.6 \pm 18.7$ & 0.41 \\
\hline WOMAC function, 2 yr postop & $65.9 \pm 26.2$ & $94.4 \pm 6.9$ & 0.12 \\
\hline WOMAC total, 1 yr postop & $72.5 \pm 27.0$ & $83.3 \pm 18.5$ & 0.41 \\
\hline WOMAC total, 2 yr postop & $66.7 \pm 19.8$ & $94.0 \pm 5.8$ & 0.07 \\
\hline Extension, ${ }^{\circ}, 1$ yr postop & $0.8 \pm 2.0$ & $0 \pm 0$ & 0.39 \\
\hline Extension, ${ }^{\circ}, 2$ yr postop & $1.7 \pm 2.9$ & $0 \pm 0$ & 0.42 \\
\hline Flexion, ${ }^{\circ}, 1$ yr postop & $126.7 \pm 4.1$ & $114.0 \pm 8.2$ & 0.022 \\
\hline Flexion, ${ }^{\circ}, 2$ yr postop & $110.0 \pm 21.8$ & $121.0 \pm 2.2$ & 0.47 \\
\hline KSS function, 1 yr postop & $90.7 \pm 14.3$ & $85.0 \pm 28.1$ & 0.65 \\
\hline KSS function, 2 yr postop & $66.7 \pm 28.9$ & $98.0 \pm 4.5$ & 0.20 \\
\hline KSS knee, 1 yr postop & $97.3 \pm 2.58$ & $94.8 \pm 2.9$ & 0.16 \\
\hline KSS knee, 2 yr postop & $72.7 \pm 25.7$ & $97.8 \pm 2.5$ & 0.23 \\
\hline KSS total, 1 yr postop & $186.5 \pm 16.8$ & $176.8 \pm 31.5$ & 0.56 \\
\hline KSS total, 2 yr postop & $139.3 \pm 53.5$ & $195.3 \pm 4.8$ & 0.21 \\
\hline \multicolumn{4}{|c|}{$\begin{array}{l}\text { KSS = Knee Society Score; MPA = medial parapatellar approach; SF-12 MCS = } \\
\text { Short-Form } 12 \text { Mental Composite Score; SF-12 PCS = Short-Form } 12 \text { Physical Composite } \\
\text { Score; SV = subvastus approach; WOMAC = Western Ontario and MacMaster } \\
\text { Universities Osteoarthritis Index. }\end{array}$} \\
\hline
\end{tabular}

Table 3. Outcome scores in medial parapatellar and lateral parapatellar approach patients with valgus preoperative alignment up to 1 year postoperative

\begin{tabular}{lccc} 
& \multicolumn{2}{c}{ Group; mean \pm SD } & \\
\cline { 2 - 3 } Outcome & MPA & LPA & p value \\
\hline SF-12 MCS, 1 yr postop & $46.5 \pm 17.7$ & $43.0 \pm 7.1$ & 0.73 \\
\hline SF-12 PCS, 1 yr postop & $53.0 \pm 7.1$ & $34.5 \pm 3.7$ & 0.011 \\
\hline WOMAC pain, 1 yr postop & $92.5 \pm 3.5$ & $71.7 \pm 10.4$ & 0.08 \\
\hline WOMAC stiffness, 1 yr postop & $62.5 \pm 17.7$ & $66.3 \pm 7.5$ & 0.81 \\
\hline WOMAC function, 1 yr postop & $89.0 \pm 9.9$ & $61.7 \pm 4.5$ & 0.022 \\
\hline WOMAC total, 1 yr postop & $85.0 \pm 5.7$ & $67.0 \pm 6.6$ & 0.05 \\
\hline Extension, ${ }^{\circ}, 1$ yr postop & $1.7 \pm 2.9$ & $2.5 \pm 2.9$ & 0.72 \\
\hline Flexion, ${ }^{\circ}, 1$ yr postop & $123.3 \pm 5.8$ & $110.0 \pm 11.5$ & 0.11 \\
\hline KSS function, 1 yr postop & $93.3 \pm 11.5$ & $43.8 \pm 54.4$ & 0.17 \\
\hline $\begin{array}{l}\text { KSS knee, } 1 \text { yr postop } \\
\text { KSS total, } 1 \text { yr postop }\end{array}$ & $98.3 \pm 2.1$ & $95.3 \pm 2.5$ & 0.15 \\
\hline $\begin{array}{l}\text { KSS = Knee Society Score; LPA = lateral parapatellar approach; MPA = medial } \\
\text { parapatellar approach; SF-12 MCS = Short-Form 12 Mental Composite Score; SF-12 PCS } \\
\text { = Short-Form 12 Physical Composite Score; WOMAC = Western Ontario and } \\
\text { MacMaster Universities Osteoarthritis Index. }\end{array}$ & \\
\hline
\end{tabular}

The MV approach for TKA was first described as a modification of the SV approach to allow for easier exposure. ${ }^{3}$ Similar to the other quadriceps-sparing approaches, it offers the theoretical benefits of improved patellar tracking, decreased postoperative pain, and quicker return of quadriceps strength. ${ }^{6}$ Results in the lit- erature, however, have been mixed. Quadriceps-sparing approaches may have a higher risk of malalignment and component malposition. ${ }^{9}$ An early study by Keating and colleagues published in 1999 comparing short-term outcomes between MV and MPA found no difference in ROM, straight leg raise, extensor lag, or rehabilitation at time of discharge. ${ }^{6}$ There was a higher rate of postoperative hematoma and manipulation in the MV patients, leading the group to conclude that they could not recommend this approach. Subsequent studies have been more promising. A recent meta-analysis of 32 randomized controlled trials showed that MV patients had significantly lower pain scores at 2 weeks postoperative than MPA patients, but found no difference at other time points. ${ }^{17}$ Range of motion was also significantly greater at 1 week postoperative for the MV group, but there was no difference at other time points. Midvastus approaches took significantly longer in terms of surgical time than MPA. There was also no difference in KSS, time to straight leg raise, intraoperative blood loss, length of hospital stay, or postoperative complications. ${ }^{17}$

In our cohort of MV patients, we found no significant difference in SF-12, WOMAC, ROM, or KSS scores at 1 and 2 years postoperative. This was consistent with the results of prior studies, which tend to show short-term benefits of quadriceps-sparing approaches, but no difference in long-term outcomes. ${ }^{3,17}$

Another quadriceps-sparing approach, the SV approach was first popularized in 1991 by Hofmann, who sought out a more anatomic approach to the knee. ${ }^{5}$ Advantages of this approach include the fact that it also does not violate the extensor mechanism. Additionally, it leaves the majority of vessels supplying the patella intact, if the dissection is carried out carefully. ${ }^{1}$ Unfortunately, the SV approach is limited by difficulty with exposure and everting the patella. ${ }^{7,8}$ Currently, it is typically used only in thin patients with mobile tissues undergoing primary TKA. Randomized controlled trials comparing this approach to the MPA have reported mixed results. Some, such as that by Roysam and colleagues, reported earlier straight leg raise, reduced blood loss, lower opiate consumption, improved patellar tracking, and better knee flexion earlier in the recovery process. $^{8} \mathrm{~A}$ metaanalysis by Liu and colleagues showed that the SV approach resulted in improved ROM at 1 week postoperative $(p<0.05)$, but no significant difference at 6 weeks or later. ${ }^{17}$ The SV group also had earlier ability to straight leg raise. Otherwise, there were no significant differences in outcomes or complication rates.

Although our sample size was small, our SV patients showed some promising results. The SV group had higher SF-12 PCS $(p=0.036)$ and WOMAC stiffness scores $(p=0.014)$ at 2 years postoperative. Interestingly, our study showed long-term benefits with this quadricepssparing approach, unlike most of the existing literature. ${ }^{17}$ 
The MPA group did, however, have significantly higher flexion at 1 year postoperative $(p=0.022)$. This was quite surprising, given that prior randomized controlled trials found no difference in long-term ROM between the 2 approaches, or in the case of the work by VarelaEgocheaga and colleagues, improved ROM in SV patients at 1 year postoperative. ${ }^{13,17}$ It was surprising that the group with reduced flexion had superior stiffness scores. The WOMAC stiffness score is a subjective scale in which patients rate their stiffness from none to extreme. Although we are unsure why the SV group had higher stiffness scores yet reduced flexion, it may have to do with a discrepancy in the patients' perceived subjective stiffness and objectively measured ROM. It is also possible that patients interpreted "stiffness" as being more than just a restriction in ROM. Clinically, it is often interesting that patients' reports of stiffness do not seem to be related to their ROM.

Finally, the LPA is another alternative to the MPA, which was first described in 1982 and later popularized by Keblish in $1991 .^{4,18}$ While also technically demanding, ${ }^{4}$ LPA allows for more direct access to lateral soft tissues in valgus knees. ${ }^{1}$ Some proponents use this approach in valgus knees because they fear a standard MPA would further promote patellar maltracking. ${ }^{19}$ Additionally, the LPA leaves the medial vasculature and nervous structures undisturbed. ${ }^{1}$ Studies on the LPA unfortunately focus on valgus knees, with little data available to analyze this approach in varus or neutral knees. Furthermore, many studies incorporate use of a TTO, which makes results difficult to interpret. Nonetheless, several studies have reported promising outcomes with LPA in valgus knees. Sekiya and colleagues found improved postoperative flexion in the LPA group $(p<0.001)$, but used extensive lateral releases, including the iliotibial band in many cases. ${ }^{15}$ They found no difference in surgical duration, complications, blood loss, postoperative alignment, laxity, patient-reported outcome scores, and KSS. A recent meta-analysis also compared the LPA to the MPA for valgus knees and found improved KSS in the LPA group, but similar alignment, operative duration, blood loss, WOMAC scores, postoperative pain, and ROM between the 2 approaches. ${ }^{16}$

As the LPA is commonly described for valgus knees, allowing for direct access to the tight lateral structures, our analysis for this group involved only knees with valgus preoperative alignment. Our comparison of the MPA and LPA in valgus knees actually showed significantly lower SF-12 PCS ( $p=0.011)$ and WOMAC function scores $(p=0.022)$ at 1 year postoperative for the LPA group. There was no significant difference in other components of the SF-12, WOMAC, ROM, or KSS. This is also unlike findings from prior randomized trials, which showed no significant difference or advantage with the LPA. ${ }^{16}$ It is important to note, however, that our LPA patients tended to have a more severe preoperative valgus deformity than the MPA patients $(p=0.024)$.

\section{Limitations}

Our study did have notable limitations. Alternative TKA approaches are not typically performed by surgeons at our institution and, as such, this was a single-surgeon study with a small sample size. The study was also retrospective in nature, and patient outcome data were not collected earlier than 1 year postoperative, which prevented us from analyzing any early benefits of quadricepssparing approaches. A large, high-quality randomized trial with extended follow-up is required to compare surgical approaches for TKA.

\section{Conclusion}

Compared with a standard MPA, the MV approach shows no significant difference in outcomes up to 2 years. The SV approach shows superior SF-12 and WOMAC scores at 2 years postoperative, but worse flexion at 1 year. The LPA for valgus knees had inferior SF-12 and WOMAC scores than the MPA, but selected for a more severe preoperative valgus deformity. Ultimately, a large randomized trial with extensive followup is recommended to verify the benefits of quadricepssparing approaches to TKA.

Affiliations: From the Department of Orthopaedic Surgery, Western University, London, Ont. (S. Sidhu, Somerville, A. Sidhu, Willing, Teeter, Lanting).

Competing interests: R. Willing is a paid consultant for Lima Corp. and receives research support from Stryker, outside the submitted work. M.Teeter reports stock in IdealFit Spacer Technologies and is a member of the advisory boards of the International Society for Technology in Arthroplasty and the Canadian RSA Network.

Contributors: S. Sidhu, M. Teeter and B. Lanting designed the study. S. Sidhu, L. Somerville and A. Sidhu acquired the data, which S. Sidhu, R. Willing and B. Lanting analyzed. S. Sidhu and B. Lanting wrote the article, which all authors reviewed. All authors gave final approval of the article to be published.

Content licence: This is an Open Access article distributed in accordance with the terms of the Creative Commons Attribution (CC BYNC-ND 4.0) licence, which permits use, distribution and reproduction in any medium, provided that the original publication is properly cited, the use is noncommercial (i.e., research or educational use), and no modifications or adaptations are made. See: https://creativecommons. org/licenses/by-nc-nd/4.0/

\section{References}

1. Vaishya R, Vijay V, Demesugh DM, et al. Surgical approaches for total knee arthroplasty. 7 Clin Orthop Trauma 2016;7:71-9.

2. Hofmann AA, Tkach TK, Evanich CJ, et al. Posterior stabilization in total knee arthroplasty with use of an ultracongruent polyethylene insert. 7 Arthroplasty 2000;15:576-83.

3. Engh GA, Holt BT, Parks NL. A midvastus muscle-splitting approach for total knee arthroplasty. 7 Arthroplasty 1997;12:322-31. 
4. Keblish PA. The lateral approach to the valgus knee. Surgical technique and analysis of 53 cases with over two-year follow-up evaluation. Clin Orthop Relat Res 1991;52-62.

5. Hofmann AA, Plaster RL, Murdock LE. Subvastus (Southern) approach for primary total knee arthroplasty. Clin Orthop Relat Res 1991;70-7.

6. Keating EM, Faris PM, Meding JB, et al. Comparison of the midvastus muscle-splitting approach with the median parapatellar approach in total knee arthroplasty. 7 Artbroplasty 1999;14:29-32.

7. Matsueda M, Gustilo RB. Subvastus and medial parapatellar approaches in total knee arthroplasty. Clin Orthop Relat Res 2000;161-8.

8. Roysam GS, Oakley MJ. Subvastus approach for total knee arthroplasty: a prospective, randomized, and observer-blinded trial. 7 Arthroplasty 2001;16:454-7.

9. Yuan FZ, Wang SJ, Zhou ZX, et al. Malalignment and malposition of quadriceps-sparing approach in primary total knee arthroplasty: a systematic review and meta-analysis. 7 Orthop Surg Res 2017;12:129.

10. Cho KY, Kim KI, Umrani S, et al. Better quadriceps recovery after minimally invasive total knee arthroplasty. Knee Surg Sports Traumatol Arthrosc 2014;22:1759-64.

11. Heekin RD, Fokin AA. Mini-midvastus versus mini-medial parapatellar approach for minimally invasive total knee arthroplasty: outcomes pendulum is at equilibrium. 7 Artbroplasty 2014;29:339-42.
12. Pan WM, Li XG, Tang TS, et al. Mini-subvastus versus a standard approach in total knee arthroplasty: a prospective, randomized, controlled study. F Int Med Res 2010;38:890-900.

13. Varela-Egocheaga JR, Suarez-Suarez MA, Fernandez-Villan M, et al. Minimally invasive subvastus approach: improving the results of total knee arthroplasty: a prospective, randomized trial. Clin Orthop Relat Res 2010;468:1200-8.

14. Varnell MS, Bhowmik-Stoker M, McCamley J, et al. Difference in stair negotiation ability based on TKA surgical approach. 7 Knee Surg 2011;24:117-23.

15. Sekiya $H$, Takatoku $\mathrm{K}$, Takada $\mathrm{H}$, et al. Lateral approach is advantageous in total knee arthroplasty for valgus deformed knee. Eur 7 Orthop Surg Traumatol 2014;24:111-5.

16. $\mathrm{Xu} \mathrm{G}, \mathrm{Fu} \mathrm{X}$, Tian $\mathrm{P}$, et al. The lateral and medial approach in total arthroplasty for valgus knee: a meta-analysis of current literature. $\mathcal{F}$ Comp Eff Res 2020;9:35-44.

17. Liu HW, Gu WD, Xu NW, et al. Surgical approaches in total knee arthroplasty: a meta-analysis comparing the midvastus and subvastus to the medial peripatellar approach. 7 Arthroplasty 2014;29:2298-304.

18. Cameron HU. The Patella in Total Knee Arthroplasty. In: Laskin RS, editor. Total Knee Replacement. Spinger; 1991:199-210.

19. Keblish PA. The lateral approach for total knee arthroplasty. 7 Knee Surg 2003;16:62-8. 